\title{
Combining digital image correlation with X-ray computed tomography for characterization of fiber orientation in unidirectional composites
}

Mahoor Mehdikhani ${ }^{1}$, Christian Breite, Yentl Swolfs, Martine Wevers, Stepan V. Lomov, Larissa Gorbatikh KU Leuven, Department of Materials Engineering, Kasteelpark Arenberg 44, 3001, Leuven, Belgium

\begin{abstract}
We apply Digital Image Correlation (DIC) to X-ray Computed Tomography (CT) slices for characterization of individual fibers misalignment in continuous fiber composites. This is an atypical application of DIC since the input is slices of a CT tomogram acquired at one time step, whereas the input of the typical deformation analysis application is $2 \mathrm{D}$ images captured over consecutive loading steps. The methodology is demonstrated and validated on synchrotron CT images of a glass/epoxy laminate, where individual fibers can be clearly identified. It is found that the average/standard deviation of the DIC displacement field in every CT slice is a good estimation of the average/standard deviation of the actual displacement of all fibers' cross-sections in that slice. This provides a shortcut to the measurement of the moments of fiber orientation distribution, compared to the algorithms that reconstruct the fiber center lines. Using this shortcut, the fiber misalignment was also characterized in synchrotron CT images of a carbon/epoxy laminate, where individual fibers were not clearly observed and where, fiber segmentation tools failed to characterize the fibers. Furthermore, being applied to synchrotron images acquired during insitu tension, the methodology is employed to quantify the evolution of misalignment during tension. The investigated 3D volumes are provided in [1] and described in [2].
\end{abstract}

Keywords: A. Fibers, D. CT analysis, D. Microstructural analysis, Digital image correlation

\section{Introduction}

Fiber misalignment, as a type of manufacturing defect or variability, degrades the mechanical properties of unidirectional (UD) Fiber-Reinforced Composites (FRCs), especially the tensile modulus, by decreasing the contribution of fibers to load carrying, and the compressive strength, through initiation of a kink band [3]. It has been noted that $15^{\circ}$ of misalignment leads to nearly $60 \%$ reduction in the tensile modulus [4] and $40 \%$ decrease in the compressive strength of glass/epoxy composites [5]. However, for some special properties such as fracture toughness, this defect can be beneficial [6]. Different techniques have been developed for

\footnotetext{
${ }^{1}$ Corresponding author (mahoor.mehdikhani@kuleuven.be)
} 
measurement of fiber orientation over the past four decades. These techniques are based on image processing and allow either $2 \mathrm{D}$ or $3 \mathrm{D}$ analysis.

The first methods used optical microscopy to acquire images for analysis. Yurgartis [3] estimated the misalignment of individual carbon fibers inside a composite through measuring the axes of their (supposedly) transverse cross-sections that appear as ellipses in almost-transverse optical micrographs (with $5^{\circ}$ deviation from the ply nominal orientation). Despite its easy applicability that has made it popular in other studies and a starting point for more advanced approaches, e.g. in [7-9], this technique suffers from low accuracy for small misalignment angles, the sensitivity to irregular fiber shapes, and the error caused by variable fiber diameter (if one value is to be assumed for all fibers for the sake of computation time and simplicity) [7]. Furthermore, it is not possible to capture the 3D misalignment with this method, and the assumption of straight longitudinal fiber sections disregards any potential waviness of individual fibers.

Analysis of fiber orientation can be performed using longitudinal micrographs of composites as well. Creighton et al. [10] developed a multiple-field image analysis method for measuring the out-of-plane misalignment, where fibers are identified as elongated features in the longitudinal micrographs. The authors tried the methodology on carbon/epoxy composites and compared the results with those obtained using the Yurgartis's [3] technique. It can be concluded that the Yurgartis's [3] technique captures the local misalignment better, while the method of Creighton et al. [10] provides a better representation of fiber misalignment in a large volume. The reason is that the former technique is applicable only at resolutions where the fiber cross-sections are clearly distinguishable, which limits the number of fibers in the field of view, while the latter technique works as long as traces of fibers can be observed in longitudinal sections. The Creighton et al. [10] method is quite fast, but it averages the orientations over square domains, and thus, does not provide any data on individual fiber orientation. Additionally, it is sensitive to surface scratches.

With a similar approach, but using the Fourier transformation and a least squares method, Kratmann et al. [11] could estimate the standard deviation of orientation within each domain, in addition to the domain average. Using software-generated micrographs, they noted that both methods have a precision better than $\pm 0.1^{\circ}$ in estimating the average fiber orientation. Yet, the Kratmann et al. [11] technique is sensitive to polishing and image quality, similarly to the method of Creighton et al. [10].

2D analysis does not yield any information on the variation of misalignment along the fibers, i.e. waviness. Microscopy also allows 3D analysis, but with low accuracy and efficiency. For example, Paluch [12] performed manual sectioning and optical microscopy along the fibers, and then, tried to reconstruct the 
spatial distribution of UD carbon fibers in 3D by pattern matching through the acquired micrographs. This allows estimation of the local fiber orientation in 3D. Although a successful first trial, this method is very time consuming and low in accuracy due to the error associated with matching the fibers cross-section in successive sections and in determining the true depth of material removed between successive sections. As an alternative, confocal laser scanning microscopy can be used for acquiring the $3 \mathrm{D}$ images, as performed by Clarke et al. [7]. Similarly to Paluch [12], they tracked the fibers center by pattern matching. On the downside, the confocal laser microscopy is low in accuracy and not applicable to opaque materials such as carbon fiber composites.

Recently, X-ray Computed Tomography (CT) has enabled acquisition of high-resolution 3D images of FRCs easily down to the fiber scale. Although fiber bundles or individual fibers are visible in X-ray CT images, quantification of fiber orientation in the images requires post-processing. In this regard, Straumit et al. [13] developed an image processing tool, called VoxTex, that measures fiber orientation by calculating the structural tensor of the X-ray CT images. Applied to different materials, including VARTM and autoclaved carbon/epoxy composites [14], this method is very efficient and rather accurate in estimations of fiber orientation distributions, but it does not reconstruct trajectories of the fibers. Emerson et al. [15] developed an interactive segmentation tool, named Insegt Fibre, which detects single fibers in high-resolution X-ray CT images using a dictionary-based probabilistic method, which allows determination of local fiber misalignment. Despite being very efficient and accurate in estimation of fiber trajectories, this technique needs an additional step of defining a dictionary of the given fiber cross-section.

Nikishkov et al. [16] proposed an algorithm to generate a finite element mesh, of which the element edges follow the local orientation of fibers. The local fiber orientation was identified from longitudinal X-ray CT slices of fiber bundles, at the mesoscale, using an edge detection technique. Thus, the resolution of the orientation analysis could not be finer than the fiber bundle thickness. With a similar approach, Krumenacker et al. [17] estimated fiber waviness after segmenting individual fibers via a manually trained Fast-RandomForest algorithm in X-ray CT images of a vacuum bag-only carbon/epoxy corner laminate. After segmentation, the images were registered (overlaid on each other) and warped (to ensure that measurements in curved regions are performed with respect to exact reference axes and planes), and then the fibers were skeletonized into line-segments, of which the orientation could be measured.

Yoshimura et al. [18] proposed a different approach for processing of the X-ray CT images. They used, for the first time to our best knowledge, Digital Image Correlation (DIC) to characterize the fiber orientation 
in the successive image slices of a carbon/epoxy laminate. They could estimate average and standard deviation of fiber orientation, which were in a reasonable agreement with manually measured values. However, the low fiber diameter-to-pixel size ratio, i.e. 2.5, did not allow characterization of the local orientation of individual fibers. Bernasconi et al. [19] used the same idea (using DIC) to measure the fiber orientation in short fiber reinforced composites. They proposed a procedure, based on identification of local coordinate system for fiber segments via DIC, to overcome the challenge in tracking short fibers in consecutive CT slices. Amjad et al. [20] used DIC to estimate the orientation of continuous fibers in optical images of ceramic-matrix and carbon-fiber polymer-matrix composites. Although they developed a highefficiency method for tracking individual fibers with DIC, their image acquisition includes automated mechanical polishing, preparing each section for automatic acquisition of hundreds of micrographs, and digitally stitching the micrographs, which is cumbersome and prone to preparation and imaging artefacts. That is why the authors suggest applying DIC orientation analysis to CT images as future work.

The present study performs a DIC-based characterization of fiber misalignment in X-ray CT tomograms of continuous FRCs, which would be applicable to both low- and high-resolution images (low and high fiber diameter-to-pixel size ratio). More specifically, the grayscale pattern in CT slices is used by DIC to track the fiber cross-sections, and consequently, to quantify the local misalignment of the fibers. This is a special application of DIC since DIC is typically applied to a set of 2D images taken over consecutive time steps to quantify the deformation, while here, it is applied to a 3D image taken at a single time step to quantify the variability in the material structure. The methodology is examined on synchrotron CT tomograms of two materials: glass/epoxy laminate, with well-distinguishable individual fibers in the scans and carbon/epoxy laminate, with less distinct fibers in the scans. The results are verified against two established CT postprocessing tools. Once validated, the methodology is employed to quantify the evolution of fiber misalignment during tensile loading. For each material, the local misalignment analysis is performed on a set of volumes, acquired in-situ at different steps during tensile loading of the material. The investigated tomograms are provided in [1] and described in [2].

\section{Composite specimen, loading, and image acquisition}

Two composite materials are investigated: 1) a cross-ply $\left[90_{9} / 0_{9}\right]_{s}$ glass/epoxy laminate, of which the reinforcement is PPG Hybon 2026 glass fibers with nominal fiber diameter of $15 \mu \mathrm{m}$, and 2) a cross-ply $\left[90_{4} / 0_{5}\right]_{\mathrm{s}}$ carbon/epoxy laminate with Mitsubishi $34-700 W D$ carbon fibers with a $7-\mu \mathrm{m}$ nominal diameter. Both fibers were impregnated (separately) with 736LT epoxy by North Thin Ply Technology. The laminates 
were stacked with hand lay-up. Having the same matrix material, both laminates were cured for $60 \mathrm{~min}$ at 70 ${ }^{\circ} \mathrm{C}$ and for 45 minutes at $120{ }^{\circ} \mathrm{C}$ temperature and $0.5 \mathrm{MPa}$ pressure inside an autoclave, with a -0.7 bar vacuum pressure. The cured laminate thickness is $0.9 \mathrm{~mm}$ for glass/epoxy and $1 \mathrm{~mm}$ for carbon/epoxy.

A double-edge notched specimen (Fig. 1) with a notch section area of $\sim 1 \times 1 \mathrm{~mm}^{2}$ was cut from each laminate with waterjet machining, similar to the specimens in [21]. Being scanned via synchrotron radiation, each specimen was in-situ loaded in $0^{\circ}$ tension, via a displacement-controlled loading rig provided by INSA Lyon, with a deformation rate of $\sim 1.5 \mu \mathrm{m} / \mathrm{s}$. The tests were conducted at the TOMCAT beamline of the Swiss Light Source $(S L S)$ in Villigen, Switzerland. The scans were made continuously with a propagation distance of $60 \mathrm{~mm}$, a beam energy of $20 \mathrm{kV}$, and 1000 projections with a 9-ms exposure time. The first (undeformed) scan of the glass/epoxy material was made with 2500 projections with a 30 -ms exposure time, before the loading started, while the first scan of the carbon/epoxy material was made when the loading just started (with the abovementioned settings). The voxel size was $1.1 \mu \mathrm{m}$, and the magnification was $10 \times$. The acquired projections were reconstructed into 3D volumes using an absorption-based algorithm provided by $S L S$.

A slight sample rotation $\left(<10^{\circ}\right)$ around the scanning rotation axis $(z$-axis $)$ was noted in the scanned volumes. Therefore, the volumes were rotated (using bicubic interpolation in MATLAB) so that the image coordinate system becomes the same as the laminate coordinate system: $z$-and $y$-axes are aligned with the nominal $0^{\circ}$ and $90^{\circ}$ lamina directions, respectively, $x$-axis is normal to the lamina (see Fig. 1). This ensures that the measured misalignment values will correspond to the actual misalignment of fibers. For each material, four volumes were selected, which correspond to the steps with approximately $0,40,73$, and $95 \%$ of the failure load. The $0 \%$ step volume for the carbon/epoxy material was acquired when the loading had been just started. The volumes of each material were registered (overlaid) on each other in the Avizo 2019.3 software, using 3D rigid transform and with "Normalized Mutual Information" and resampled with "Standard" (linear) interpolation. This removes any possible rigid body motion and rotation and allows easily locating the smaller Volume Of Interests (VOIs).

For the orientation analysis, a VOI of $420 \times 1030 \times 450 \mu \mathrm{m}^{3}(x, y, z)$ for the glass/epoxy and of $390 \times 830$ $\times 440 \mu \mathrm{m}^{3}(x, y, z)$ for the carbon/epoxy material was chosen in their $0^{\circ}$ plies (as indicated with a blue box in Fig. 1a and b, respectively). They are, hereafter, referred to as "glass VOI" and "carbon VOI", respectively. The $x y$ dimension of VOIs are defined such that it covers most of the unnotched volume in the $0^{\circ}$ plies of each material. The glass VOI contains around 1100 fibers, but the exact number of fibers in the carbon VOI cannot be determined due to the low diameter-to-pixel size ratio. The number of fibers is estimated to be 
4500 using a fiber volume fraction of $48 \%$. The VOIs were cropped from the large volumes. Except the first (undeformed) volume of the glass/epoxy material that was scanned with more and longer projections, the other volumes are rather noisy. Hence, those VOIs were filtered with a 3D "non-local means" algorithm in Avizo 2019.3 with a search window of 10 px and local neighborhood of 3 px. A "Ring Artifact Removal" filter was also applied to the volumes in Avizo before applying the non-local means filter. The VOIs are presented in [2].

\section{DIC orientation measurement methodology}

DIC is a full-field technique, developed primarily to quantify the deformation in images of the surface of a deforming material. This is achieved by tracking the grayscale pattern, which is provided by a natural or artificial random speckle pattern in the images. For ply-level deformation analysis in FRCs, the fiber crosssections can serve as an adequate speckle pattern [22], while for specimen-level and interfiber-level analysis, an artificial random speckle pattern is required, such as in [22] and [23, 24].

In contrast with its typical application, in the current study, DIC is employed to characterize the orientation of fibers in an X-ray tomogram. Thus, DIC is not used to measure deformation of an object during loading but to characterize the variability in the microstructure of a non-loaded material. This is achieved by applying DIC to the transverse slices of a 3D volume, as presented in Fig. 2a. DIC treats the slices as if they are images taken from a deforming object over time, and hence, tracks the changes in their grayscale pattern. Tracking the pattern, which in this case is created by (segments of) the fiber cross-sections, in every slice allows DIC to quantify the location and, consequently, the misalignment of every fiber through the whole volume, as shown schematically in Fig. 2 b.

DIC calculates two displacement vectors, one horizontal (u) and one vertical (v), for every point in every transverse slice, making up two displacement fields (Fig. 2a). A MATLAB script was written to extract from the fields the corresponding displacements of every individual fiber and translate them to local misalignment and to global orientation (Fig. 2b). For each slice, the script:

1) detects the fibers using the MATLAB circle detection function (imfindcircles) and identifies the $x$ and $y$ coordinates of their centers,

2) finds the closest points in the DIC resulting field to the fiber centers, and

3) assigns the corresponding $\mathbf{u}$ and $\mathbf{v}$ in the resulting field to every circle center.

Therefore, every fiber in every slice is assigned with two displacement vectors, $\mathbf{u}$ and $\mathbf{v}$, which correspond to their out-of-plane (green) and in-plane (blue) local misalignment, as respectively indicated in 
Fig. 2b. The plane refers to the plane of the laminate. The script can further estimate the local in-plane $(\alpha)$ and out-of-plane $(\beta)$ orientation for every fiber, using the following formulas, where $l$ is the distance between two consecutive slices, and $u$ and $v$ are respectively the lengths of the vectors $\mathbf{u}$ and $\mathbf{v}$ :

$$
\begin{aligned}
& \alpha=\tan ^{-1}\left(\frac{u}{l}\right) \\
& \beta=\tan ^{-1}\left(\frac{v}{l}\right)
\end{aligned}
$$

The global orientation of each fiber can be estimated using the slope of a linear fit to their location at each slice (explained in Section 4.1.1). It needs to be emphasized that the in-plane and out-of-plane displacements correspond to the change in the location of the fiber cross-section across the consecutive slices and not to the deformation of the material. Deformed DIC coordinates are used for the analysis, meaning that the $x$ and $y$ coordinates of the data points in the resulting DIC field for each slice are changed based on the displacements occurred in the preceding slice. Assigning displacement values to every fiber is feasible only when fibers are clearly distinguishable in CT images. However, a shortcut approach is proposed (in Section 4.1.1) which enables estimation of the average misalignment of fibers even in images where fibers are not clearly visible.

DIC was performed using VIC-2D 6 software (Correlated Solutions). Since some fibers have significant change of their location through the whole volume, incremental correlation was employed, which correlates each image with the one before (and not with the first reference image). The size of the DIC searching window, or subset, was set to $19 \mathrm{px}(\approx 21 \mu \mathrm{m})$, and the step size, i.e. the shift of two successive subsets, was set to $1 \mathrm{px}(\approx 1.1 \mu \mathrm{m})$. Using the pattern made by the fiber cross-sections as the required random speckle pattern for DIC, these values were found to be a trade-off between the DIC ability to correlate the images and the accuracy of the displacement fields. If subsets are too small, they cannot contain enough grayscale pattern and thus the correlation fails, and on the other hand, if they are too large the effect of displacement averaging over one subset will be significant, which leads to a high underestimation of displacement. Scaled-to-size subsets are shown on the microstructure of both materials in Fig. 1. The correlation is performed using Gaussian subsets, Optimized 6-tap interpolation, and Normalized squared differences criterion. The methodology is first tried on the undeformed VOI of the glass/epoxy material (Fig. 1a), and once validated, on the deformed VOIs. It is also applied to the carbon/epoxy VOIs

\section{Results and discussion}

\subsection{Glass/epoxy material}


Fig. 3a and c represent the DIC full-field displacements of the last slice (as an example) of the undeformed glass VOI. The fields are inhomogeneous, which means that in each slice, fibers have different $u$ and different $v$ values, signifying dissimilar local misalignments. Both positive and negative values of $u$ and $v$, which signify both out-of-plane and in-plane misalignment, are observed. Fig. $3 \mathrm{~b}$ and d show, respectively, the DIC-resulting $v$ and $u$ for the detected fibers in the same last slice, processed in MATLAB. Clusters of fibers with similar in-plane misalignment are visible in Fig. 3a and b. The displacement vector of each detected fiber along with the location of the fiber in the last slice are presented in Fig. 3e, where clustered misalignment of fibers can be noticed.

\subsubsection{Misalignment characterization in undeformed VOI}

One fiber analysis: To perform a preliminary verification, misalignment of one fiber is investigated. A fiber in the undeformed glass VOI is selected, as indicated in Fig. 4a. Its in-plane and out-of-plane DIC displacements in each slice, measured with regard to the first reference slice, are plotted in Fig. 4b. The $v$ and $u$ displacements of the fiber center in the last slice (Slice B) with regard to its position in the first slice (Slice A) are also manually measured. The manually measured $v$ and $u$ are 18.7 and $4.4 \mu \mathrm{m}$, while the DIC resulting values, being the last values in Fig. $4 \mathrm{~b}$, are 18.3 and $3.9 \mu \mathrm{m}$, which have a relative error of respectively $2.1 \%$ and $11.3 \%$ compared to the manual values. Fig. $4 \mathrm{~b}$ also shows that the displacement of the fiber cross-section between two consecutive slices increases almost linearly, meaning that the fiber is nearly straight (but has an angle of $2.4^{\circ}$ with the nominal $0^{\circ}$ direction, i.e. $z$-axis).

Thanks to incremental correlation, no limits on maximum detectable misalignment could be found, at least in this VOI. However, for larger misalignments, the difference between DIC and manual measurements increases. The maximum fiber displacement in the last slice with regard to the first slice is around $75 \mu \mathrm{m}$, which was also detected by DIC, but with an underestimation of around $30 \mu \mathrm{m}$. It should be noted that the difference of $30 \mu \mathrm{m}$ occurs at the last slice (with a distance of $440 \mu \mathrm{m}$ from the reference slice), and decreases linearly when moving towards the reference slice. Moreover, performing non-incremental DIC, it was found that once the fiber displacement, calculated via direct correlation to the first slice (and not to the preceding slice), exceeds $\sim 4 \mu \mathrm{m}$, the correlation fails. Assuming a 1.1- $\mu \mathrm{m}$ spacing between the two slices (while the real distance is $60 \mu \mathrm{m}$ ), the displacement of $4 \mu \mathrm{m}$ translates to a misalignment of $\sim 75^{\circ}$. This is an unrealistic case for consecutive slices, but has an important implication when it comes to excluding some slices, e.g. for reducing analysis time: the spacing between the selected slices should be low enough to avoid 
fiber displacements above $4 \mu \mathrm{m}$. Of course, these numbers are valid for the current case, with a fiber nominal diameter of $15 \mu \mathrm{m}$, voxel size of $1.1 \mu \mathrm{m}, 10 \times$ magnification, and subset size of $21 \mu \mathrm{m}$.

Fibers' displacement across consecutive slices: The nearly linear increase of the displacement of fiber cross-sections between consecutive slices is also noticed when the average displacement of all fibers in every slice (measured with regard to the first reference slice) were plotted versus the slice $z$-coordinate, in Fig. 5a (DIC_fibers linear fit). The linear increase is further explored by evaluating the linearity of the displacement increase for every fiber in the VOI. Thus, the linear correlation coefficient of displacement versus the slice $z-$ coordinate is computed for every fiber. When a linear correlation coefficient of 0 signifies no linearity and 1 corresponds to a perfectly linear relationship, it is found that the average coefficient is 0.84 for in-plane and 0.29 for out-of-plane displacement. The percentage of fibers with a linear correlation coefficient larger than 0.7 is $89 \%$ for in-plane displacement and about $52 \%$ for out-of-plane displacement. The high correlation coefficient for most of the fibers, especially for out-of-plane displacement, suggests that the majority of fibers are quite straight, and that they have a nearly constant misalignment across the consecutive slices (with a spacing of $1.1 \mu \mathrm{m})$.

Misalignment level: to quantify fiber alignment, the displacement increase over the slices (with $1.1 \mu \mathrm{m}$ spacing) should be investigated. It is found that $93 \%$ of fibers have increasing in-plane displacement through more than half of the slices (= half of their length), while this ratio is $64 \%$ for the out-of-plane displacement. The average increase of in-plane displacement for each fiber over all the 410 slices is averaged for all fibers, which results in a value of $0.043 \mu \mathrm{m}$, while it is $0.005 \mu \mathrm{m}$ for the out-of-plane displacement. The high (linear) increase of in-plane displacement through successive slices implies that the majority of (straight) fibers are misaligned with a similar in-plane angle from the nominal $0^{\circ}$ direction (i.e. from the $x z$-plane). This systematic misalignment means that the $0^{\circ}$ plies are tilted around the $x$-axis with regard to the plane normal to the synchrotron beam, which could have occurred during laying up, cutting, or mounting the specimen. The tilt angle is estimated below.

Fiber Orientation Distribution (FOD): Assuming that most fibers are straight, their orientation can be estimated with the slope of the line that fits their location (or alternatively their displacements) through successive slices. For example, the slope of the linear fittings to the blue and green data points in Fig. $4 \mathrm{~b}$ equals, respectively, the in-plane and out-of-plane orientation of that specific fiber, as schematically shown in Fig. 2b. These slopes are calculated for all fibers in the VOI, and the resulting FOD is plotted in Fig. 6a and b (DIC_fibers linear fit). The average of the in-plane orientation is $2.27^{\circ}$ and the standard deviation is $1.53^{\circ}$. 
The shift of the average from $0^{\circ}$, noted in Fig. $6 \mathrm{a}$, confirms that most fibers are misaligned with regard to the $x z$-plane. In fact, the $0^{\circ}$ plies have a tilt of $\sim 2.27^{\circ}$ around the $x$-axis with regard to the plane normal to the synchrotron beam. The average of the out-of-plane orientation is $0.29^{\circ}$ with a standard deviation of $0.77^{\circ}$, indicating that the $0^{\circ}$ plies are well aligned with the $y z$-plane. Although less fibers have a linear increase of their out-of-plane displacement (compared to their in-plane displacement), this approach of estimating the orientation based on linear fitting provides a reasonable level of accuracy since, even for those fibers with less linearity, the linear fit provides an overall estimation of their orientation over a given length.

Enhancing the methodology's efficiency: The technique will be much more efficient if post-processing of the DIC results for every individual fiber can be skipped. This is explored by comparing the average and standard deviation of the entire DIC displacement fields (DIC_field average) with those of the fiber displacements, in every slice. As noted in Fig. 5, the values calculated for individual fibers and the entire displacement field agree well with each other. This implies that the DIC displacement average/standard deviation in each slice is a good estimation of the average/standard deviation of displacement of all fibers in that slice. Therefore, the moments of FOD can be estimated much more efficiently, and still with a reasonably high accuracy, by just averaging the DIC displacement in every slice, and using the resulting average and standard deviation. The slightly higher values, in Fig. 5, for the individual fiber analysis compared to the entire field averages is most likely due to inclusion of the matrix regions, with no misalignment, in the latter.

Representative Volume Element $(R V E)$ : Estimating a minimum size for a suitable RVE for misalignment analysis will be useful for further investigations of misalignment in FRCs. First, 40- $\mu$ m-wide vertical and horizontal strips, displayed in Fig. 7c, are used for analysis of misalignment variation along the width and length of the slice. These strips can accommodate 1-3 fiber centers along their width and are fine enough to represent any misalignment trend. As can be noted in Fig. 7, which presents the average displacements over the strips in the last slice, the misalignments seem to be, in general, higher at the ply mid-thickness and midwidth; only the in-plane misalignment seems to increase along the width ( $y$-direction), in Fig. 7b. The highest variation is noted for the in-plane misalignment along the thickness ( $x$-direction), in Fig. 7a, which may arise from the imperfect in-plane alignment of the plies during hand lay-up. This inhomogeneity poses a challenge to determining a minimum RVE size. To deal with this issue, windows of different sizes within 50-300 $\mu \mathrm{m}$ are selected at three different locations along the width, as displayed in Fig. 8. The average and standard deviation of the displacements in these windows are presented in Fig. 8b-e. The in-plane displacement 
decreases when the window size increases. This trend does not seem to be there for the out-of-plane displacement. The curves do not change significantly anymore for windows larger than 200-250 $\mu \mathrm{m}$, per side. Therefore, a window with a $200-\mu \mathrm{m}$ side can be considered a suitable RVE.

Additionally, correlations between misalignment and other parameters are explored. No correlation could be established between misalignment and fiber radius (estimated via the MATLAB circle detection function) and between in-plane and out-of-plane misalignments.

\subsubsection{Verification versus existing tools}

The measured displacements and FODs are compared with those calculated via the Insegt Fibre fibersegmentation technique [15], developed at the Technical University of Denmark. After training the algorithm with a few examples of fiber cross-sections, Insegt Fibre detects individual fibers in the whole volume. Therefore, the coordinates of the center of every fiber cross-section in every slice are determined, which allows determination of local misalignment of fibers. The same undeformed glass VOI is analyzed with Insegt Fibre. The resulting average and standard deviation of displacement of all fibers in each slice are plotted against the slice $z$-coordinate in Fig. 5 (Insegt_fibers linear fit). There is a good agreement between the Insegt Fibre graphs and those obtained via the DIC methodology, especially for the in-plane displacement. The small underestimation with DIC, noted in the standard deviation graphs, is most likely due to the DIC displacement averaging over subsets. Moreover, using the slope of the linear fit to the fiber locations estimated with Insegt Fibre, in-plane and out-of-plane FODs are computed and plotted in Fig. 6c and d (Insegt_fibers linear fit). These graphs look similar to the corresponding DIC results, presented in Fig. 6a and $\mathrm{b}$. A more objective comparison between these distributions has been made at the end of the current section, using statistical tests, of which the results are reported in Table 1.

A more detailed analysis of fiber orientation is made using the Insegt Fibre data. Performing a nearest neighbor search on the fiber centers, calculated in Insegt Fibre, trajectories for every fiber are generated, which are smoothed via averaging 10 adjacent data points $(=11 \mu \mathrm{m})$ to reduce noise from the segmentation. Then, the fiber trajectories are sampled with splines of $11 \mu \mathrm{m}$, using a piecewise cubic Hermite interpolating polynomial in MATLAB. The Insegt Fibre algorithm tracked, smoothed, and sampled fiber trajectories are visualized with a diameter of $15 \mu \mathrm{m}$ and color coded with in-plane and out-of-plane orientations in Fig. 9. The local in-plane and out-of-plane orientations of every spline are calculated, and their statistical distribution presented in Fig. 6e and f (Insegt_spline fit), which look rather similar to the DIC-based FODs 
(Fig. 6a and b) and the Insegt-based FODs obtained via linear fitting (Fig. 6c and d). These FODs are compared in a more objective way at the end of the current section.

To evaluate the waviness of every fiber, the shortest distance of each sampled spline from the global fiber trajectory (connecting start and end point) is computed and considered as the local amplitude. By analyzing zero values for the amplitude along the fiber, the fiber is segmented into sinusoidal pieces, each representing a part of a half wave or a full half wave. Each segment is fitted with a sine function, for which the amplitude and wavelength are measured. Considering all segments of all fibers, the average amplitude is $1.33 \pm 0.93$ $\mu \mathrm{m}$, and that of the wavelength is $1129 \pm 271 \mu \mathrm{m}$. These values imply a very weak waviness over a very large length for most of the fibers. Therefore, the orientation analysis based on linear fitting can provide a proper representation for the whole composite.

Additionally, the DIC methodology is validated against the analysis of the structure tensor of the grayscale image, implemented in the VoxTex software (KU Leuven) [13]. VoxTex meshes the VOI into cubic elements of a given size and assign each element with an average orientation. Using a mesh size of $13 \mu \mathrm{m}$, the same undeformed glass VOI is analyzed. As can be noticed in Fig. 6, the resulting orientation distributions are in a very good agreement with the DIC-based FODs. The VoxTex FODs are quantitatively compared with the FODs from other techniques in the following paragraph. The resulting average and standard deviation are presented in Fig. 10, which are very close to those calculated by DIC_fibers linear fit (relative error of $0.15 \%$ for the in-plane average and $6.95 \%$ for the out-of-plane average). The VoxTex average value is the average of orientations of all the elements. The DIC field average orientations are calculated using the slope of the line passing through the first and last data points in the average/standard deviation slice displacement versus the slice $z$-coordinate (Fig. 5). In general, Fig. 10 indicates that the averages and standard deviations of fiber orientations calculated with different methods match, particularly for the in-plane case, where the averages lay within $8 \%$ from each other. Insegt Fibre measures a lower outof-plane orientation, while it calculates a higher standard deviation for both in-plane and out-of-plane orientations. The lower standard deviation for the DIC and VoxTex methods can be due to averaging the misalignment over subsets or voxels.

To compare the FODs obtained with DIC_fibers linear fit, Insegt_fibers linear fit, Insegt_spline fit, and VoxTex, three statistical two-sample tests are performed, of which the p-values are reported in Table 1. The tests are Welch's t-test, Mann-Whitney-Wilcoxon (MWW) test, and Kolmogorov-Smirnov (K-S) test, which allow comparing the means, the medians, and the entire distributions, respectively. It is noted that there is an 
excellent agreement between the DIC- and VoxTex-based FODs for both in-plane and out-of-plane cases. This proves the reliability of the DIC methodology in identifying FODs. For the in-plane case, it is found that although DIC- and Insegt-based distributions are distinct (low p-values of the K-S test), their means and medians are identical (high $p$-values of the $t$-test and MWW test, respectively). Nonetheless, for the out-ofplane case, the results of DIC and Insegt Fibre poorly match with each other. This can be due to measuring small misalignments that are below or just at the limit of the precision of the techniques. Finally, there is a nice agreement between the FODs of Insegt_fibers linear fit and Insegt_spline fit, implying that the linear fit used for determining the fiber orientations is sufficiently accurate, at least for this case.

\subsubsection{Misalignment evolution during loading}

So far, only one (undeformed) glass VOI has been analyzed. In this section, the misalignment evolution in the deformed VOIs of the glass/epoxy material, which correspond to the three loading steps, are investigated. Since the average/standard deviation of DIC displacement in each slice was found to be an adequate estimation of the average/standard deviation of displacement of all fibers in that slice, the misalignment analysis is performed using DIC slice averaging.

Fig. 11a presents, for each loading step, the average DIC in-plane and out-of-plane displacements in each slice versus the slice $z$-coordinate. The results of the fourth step are excluded since a part of the specimen had rotated due to severe damage at that load level, which makes characterization of fiber misalignment irrelevant. Fig. 11a indicates that the average in-plane displacement does not change significantly during the loading, while the average out-of-plane displacement increases slightly between Step 0 and 1 and decreases between Step 1 and 2. The increase may be due to a first random movement of fibers in the resin-rich regions in the beginning of loading, and the decrease can be attributed to a slight increase in the alignment of the fibers in the specimen with the loading direction upon increase of loading. It needs to be noted that the rigid body motion and rotation between the steps are minimized via registration of the volumes over each other.

Fig. 11b indicates that for both directions of displacement, the corresponding standard deviations reduce slightly when the loading increases. This means the fibers seem to become a bit more aligned with each other when loaded in their axial direction. Performing a basic trigonometry calculation, a misalignment of $2.14^{\circ}$ is predicted to change to $2.11^{\circ}$ with $1 \%$ of applied strain on the sample. Therefore, the misalignment change for the current case is anyway expected to be marginal and out of the precision of the investigated techniques.

The DIC average and standard deviation of fiber displacement over the loading steps are translated to orientation, using the slope of the line passing through the first and last data points of each curve in Fig 11a 
and $\mathrm{b}$ and are presented in Fig. 11c and d, along with corresponding VoxTex results. A general agreement is observed between the results of the DIC methodology and VoxTex. Except for a few cases, even the small changes in the trend of the VoxTex graphs are captured well by the DIC technique. Most of the VoxTex misalignment absolute values are larger than the corresponding DIC resulting values, which can be attributed to a slight underestimation of misalignment due to the DIC subset averaging.

The results obtained in the analysis of the misalignment evolution during loading may not be entirely representative, as: 1) only a part of the $0^{\circ}$ ply is analyzed, 2) the registration of the volume may influence slightly the measured misalignments, and 3) the damage, especially the ply splits, that happens in the last steps may lead to (partial) rotation of the ply, which affects the misalignment analysis.

It should be noted that if the specimen has a rigid body rotation, the average orientation will change, while the orientation standard deviation should not change. Nonetheless, if the rotated image is resliced according to the first principal axis, the standard deviation will change too. If the rigid body rotation is not significant, the change in the standard deviation will be negligible.

\subsection{Carbon/epoxy material}

It is observed in Fig. 12a and d that the DIC displacement fields of the last slice of the undeformed carbon VOI are inhomogeneous, meaning dissimilar misalignment of fibers through the slice. The clustering of fibers with similar in-plane misalignment is more pronounced than in the glass/epoxy material, such that even large strips of fibers with similar in-plane misalignment are observed. The very high variation in the in-plane misalignment along the thickness ( $x$-direction) can be noted in Fig. 12, where the average displacements over $40-\mu \mathrm{m}$ wide vertical and horizontal strips in the last slice are plotted. This can be due to the inaccuracy in inplane alignment of the plies during hand lay-up. Additionally, the in-plane misalignment decreases with moving toward the mid-width (in the $y$ direction) of the specimen. For this material, the analysis of individual fibers is not conducted since the individual fibers could not be well detected in MATLAB due to the small diameter-to-pixel size ratio. Therefore, the misalignment analysis is performed based on the calculation of the average and standard deviation of DIC displacement in each slice.

For the VOIs of the consecutive loading steps, the average DIC resulting in-plane and out-of-plane displacements in each slice and the respective standard deviations versus the slice $z$-coordinate are plotted in Fig. 13a and b. The absolute in-plane displacement slightly increases between Step 0 and 1 and decreases between Step 1 and 2 and between Step 2 to Step 3, which is most likely due to the alignment of the fibers with the loading direction. In general, the changes of alignment between the steps are so small that they could 
be explained just by the errors in image registration and maybe in orientation measurement. The changes in the standard deviations are more relevant since they are less sensitive to image registration. As noted in Fig. 13, the standard deviation changes are insignificant.

Average and standard deviation orientations are calculated based on the slope of the line passing through the first and last point of each curve in Fig. 13a and b and presented in Fig. 13c and d. Corresponding VoxTex results, obtained with a mesh size of $10 \mu \mathrm{m}$, are also represented in this figure. An excellent match is found between the average orientations of DIC and VoxTex. However, VoxTex estimates higher standard deviations compared to the DIC approach results, which is expected as VoxTex performs the analysis on a fine mesh while DIC, in this case, analyzes the slices. In the VoxTex results of the loaded VOIs, some extreme orientation values were found, which were deemed to be errors since such extreme levels of misalignment cannot occur with the applied loading. The number of elements with false values increased with loading and was, in general, larger for the carbon/epoxy material. For every VOI, values with a change in in-plane misalignment more than $3^{\circ}$ and/or a change in out-of-plane misalignment more than $0.5^{\circ}$ were filtered out. These values were selected as an arbitrary overestimation of the possible misalignment between two loading steps, based on the basic trigonometry estimation of misalignment change during loading (Section 4.1.3). The number of elements with false values is, anyway, negligible compared to the total number of elements.

It needs to be noted that Insegt Fibre loses full-fiber tracing capability in the images of the carbon/epoxy material due to a rather small fiber diameter-to-pixel size ratio and high amount of noise, as observed in Fig. 1b. At the moment, Insegt Fibre works best when the fibers are distinguishable in the CT slices.

\section{Concluding remarks}

We perform a DIC-based quantification of fiber orientation in CT volumes. The methodology is applied to synchrotron CT images of a glass/epoxy laminate with a voxel size of $1.1 \mu \mathrm{m}$. It is found that the fibers are almost straight. Thus, their orientation is calculated with linear fits to their location. The results are verified versus the Insegt Fibre and VoxTex techniques. Statistical tests show an excellent agreement between the DIC-based FODs and those obtained via VoxTex. Piecewise fiber orientation analysis via Insegt Fibre confirms that the linear fit provides a reasonable estimation of the locally measured FOD, in this case.

It is found that the rather time-consuming analysis of individual fibers can be bypassed by using the DIC displacement average/standard deviation in each CT slice as it provides a good estimation of the average/standard deviation of displacement of all fibers in that slice. Using this shortcut comes at the cost of a certain underestimation of misalignments due to inclusion of matrix regions with no misalignments. 
However, it allows the methodology to be even applicable to CT images, in which the fibers are not easily distinguishable. This is tried on an example of carbon/epoxy laminate, and the resulting FOD is found to be in a good match with VoxTex results. Moreover, negligible changes of misalignment with tensile loading have been noted. The entire analyzed data is supplied in [2].

The methodology is very simple to apply and is objective since it does not need any manual training of the algorithm. Furthermore, it can be used by anyone who has access to a DIC software. Another advantage over the two existing tools is that this DIC-based methodology can be used for individual fiber misalignment analysis, if the fibers are detectable in the images (not possible via VoxTex), as well as for estimation of local average misalignment, even when the fibers are not clearly detectable (not possible via Insegt Fibre). Of course, the individual fiber analysis is more time consuming. As a drawback, DIC subset averaging can lead to underestimation of misalignments. The underestimation is expected to be more significant for lower images resolutions. This can be diminished by using smaller subsets, of course up to the point that the subsets still contain enough grayscale variability to build recognizable patterns.

\section{Acknowledgments}

The study is funded by the KU Leuven Research Council, project C24/17/052 and FWO Postdoc Fellowship, project ToughImage $(1263421 N)$. It is also supported by the European Union's Horizon 2020 research and innovation program, Marie Skłodowska-Curie grant agreement No. 722626 (FiBreMoD project). The authors acknowledge the Paul Scherrer Institut, Villigen, Switzerland for provision of synchrotron radiation beamtime at the beamline TOMCAT of the SLS, proposal No. 20161157. Support to optimize the acquisition settings of the beamline was provided by I. Sinclair from University of Southampton and C. Schlepütz from SLS. The in-situ testing rig was kindly provided by E. Maire from INSA Lyon. We deeply thank F. Mesquita for the assistance on sample preparation and image acquisition and M.J. Emerson (DTU) for the hands-on training provided for Insegt Fibre. The support of the KU Leuven XCT Centre is acknowledged. S.V. Lomov holds Toray Chair for Composite Materials at KU Leuven, support of which is gratefully acknowledged too.

\section{References}

1. A dataset of micro-scale tomograms of unidirectional glass fiber/epoxy and carbon fiber/epoxy composites acquired via synchrotron computed tomography during in-situ tensile loading. 2020: Mendeley Data. 
2. Mehdikhani, M., C. Breite, Y. Swolfs, M. Wevers, S.V. Lomov, and L. Gorbatikh, A dataset of micro-scale tomograms of unidirectional glass fiber/epoxy and carbon fiber/epoxy composites acquired via synchrotron computed tomography during in-situ tensile loading. Data in Brief, 2020: p. 106672.

3. Yurgartis, S., Measurement of small angle fiber misalignments in continuous fiber composites. Composites Science and Technology, 1987. 30(4): p. 279-293.

4. Li, Y., B. Stier, B. Bednarcyk, J.-W. Simon, and S. Reese, The effect of fiber misalignment on the homogenized properties of unidirectional fiber reinforced composites. Mechanics of Materials, 2016. 92: p. 261-274.

5. Pinho, S., L. Iannucci, and P. Robinson, Physically-based failure models and criteria for laminated fibre-reinforced composites with emphasis on fibre kinking: Part I: Development. Composites Part A: Applied Science and Manufacturing, 2006. 37(1): p. 63-73.

6. Truss, R.W., P.J. Hine, and R.A. Duckett, Interlaminar and intralaminar fracture toughness of uniaxial continuous and discontinuous carbon fibrelepoxy composites. Composites Part A: Applied Science and Manufacturing, 1997. 28(7): p. 627-636.

7. Clarke, A., G. Archenhold, and N. Davidson, A novel technique for determining the 3D spatial distribution of glass fibres in polymer composites. Composites science and technology, 1995. 55(1): p. 75-91.

8. Kaczmarek, H., Ultrasonic detection of the development of transverse cracking under monotonic tensile loading. Composites Science and Technology, 1993. 46(1): p. 67-75.

9. Zak, G., C.B. Park, and B. Benhabib, Estimation of Three-Dimensional Fibre-Orientation Distribution in Short-Fibre Composites by a Two-Section Method. Journal of Composite Materials, 2001. 35(4): p. 316-339.

10. Creighton, C., M. Sutcliffe, and T. Clyne, A multiple field image analysis procedure for characterisation of fibre alignment in composites. Composites Part A: Applied Science and Manufacturing, 2001.32(2): p. 221-229. 
11. Kratmann, K.K., M. Sutcliffe, L. Lilleheden, R. Pyrz, and O.T. Thomsen, A novel image analysis procedure for measuring fibre misalignment in unidirectional fibre composites. Composites Science and Technology, 2009. 69(2): p. 228-238.

12. Paluch, B., Analysis of geometric imperfections in unidirectionally reinforced composites. Proc. ECCM6, Bordeaux, 1993: p. 305-310.

13. Straumit, I., S.V. Lomov, and M. Wevers, Quantification of the internal structure and automatic generation of voxel models of textile composites from X-ray computed tomography data. Composites Part A: Applied Science and Manufacturing, 2015. 69: p. 150-158.

14. Nguyen, N.Q., M. Mehdikhani, I. Straumit, L. Gorbatikh, L. Lessard, and S.V. Lomov, Micro-CT measurement of fibre misalignment: Application to carbon/epoxy laminates manufactured in autoclave and by vacuum assisted resin transfer moulding. Composites Part A: Applied Science and Manufacturing, 2018. 104: p. 14-23.

15. Emerson, M.J., K.M. Jespersen, A.B. Dahl, K. Conradsen, and L.P. Mikkelsen, Individual fibre segmentation from $3 D X$-ray computed tomography for characterising the fibre orientation in unidirectional composite materials. Composites Part A: Applied Science and Manufacturing, 2017. 97: p. 83-92.

16. Nikishkov, G., Y. Nikishkov, and A. Makeev, Finite element mesh generation for composites with ply waviness based on X-ray computed tomography. Advances in Engineering Software, 2013. 58: p. $35-44$.

17. Krumenacker, N., A. Madra, and P. Hubert, Image-based characterization of fibre waviness in a representative vacuum-bagged corner laminate. Composites Part A: Applied Science and Manufacturing, 2020. 131: p. 105774.

18. Yoshimura, A., R. Hosoya, J. Koyanagi, and T. Ogasawara, X-ray computed tomography used to measure fiber orientation in CFRP laminates. Advanced Composite Materials, 2016. 25(1): p. 1930.

19. Bernasconi, A., M. Carboni, and R. Ribani, On the combined use of Digital Image Correlation and Micro Computed Tomography to measure fibre orientation in short fibre reinforced polymers. Composites Science and Technology, 2020. 195: p. 108182. 
20. Amjad, K., W.J.R. Christian, K. Dvurecenska, M.G. Chapman, M.D. Uchic, C.P. Przybyla, and E.A. Patterson, Computationally efficient method of tracking fibres in composite materials using digital image correlation. Composites Part A: Applied Science and Manufacturing, 2020. 129: p. 105683.

21. Swolfs, Y., H. Morton, A.E. Scott, L. Gorbatikh, P.A.S. Reed, I. Sinclair, S.M. Spearing, and I. Verpoest, Synchrotron radiation computed tomography for experimental validation of a tensile strength model for unidirectional fibre-reinforced composites. Composites Part A: Applied Science and Manufacturing, 2015. 77: p. 106-113.

22. Mehdikhani, M., E. Steensels, A. Standaert, K.A.M. Vallons, L. Gorbatikh, and S.V. Lomov, Multi-scale digital image correlation for detection and quantification of matrix cracks in carbon fiber composite laminates in the absence and presence of voids controlled by the cure cycle. Composites Part B: Engineering, 2018. 154: p. 138-147.

23. Mehdikhani, M., M. Aravand, B. Sabuncuoglu, M.G. Callens, S.V. Lomov, and L. Gorbatikh, Fullfield strain measurements at the micro-scale in fiber-reinforced composites using digital image correlation. Composite Structures, 2016. 140: p. 192-201.

24. Mehdikhani, M., A. Matveeva, M.A. Aravand, B.L. Wardle, S.V. Lomov, and L. Gorbatikh, Strain mapping at the micro-scale in hierarchical polymer composites with aligned carbon nanotube grafted fibers. Composites Science and Technology, 2016. 137(Supplement C): p. 24-34.

Table 1. P-values of the two-sample Welch's t-test, Mann-Whitney-Wilcoxon (MWW) test, and KolmogorovSmirnov (K-S) test, between the FODs obtained with DIC_fibers linear fit, Insegt_fibers linear fit, Insegt_spline fit, and VoxTex - the tests allow comparing the means, the medians, and the entire distributions, respectively.

\begin{tabular}{ccccccc}
\hline & $\begin{array}{c}\text { Statistical } \\
\text { test }\end{array}$ & $\begin{array}{c}\text { DIC-fibers } \\
\text { linearfit vs. } \\
\text { VoxTex }\end{array}$ & $\begin{array}{c}\text { DIC-fibers linearfit } \\
\text { vs. Insegt_spline fit }\end{array}$ & $\begin{array}{c}\text { Insegt_fibers linear } \\
\text { fit vs. Insegt_spline } \\
\text { fit }\end{array}$ & $\begin{array}{c}\text { VoxTex vs. } \\
\text { Insegt_splinefit }\end{array}$ & $\begin{array}{c}\text { DIC-fibers linearfit vs. } \\
\text { Insegt_fibers linearfit }\end{array}$ \\
\hline $\begin{array}{c}\text { In-plane } \\
\text { FOD }\end{array}$ & t-test & 0.92 & 0.3 & 0.08 & 0 \\
0.56 & 0.55 & 0.18 & 0.08 & 0 & 0.61
\end{tabular}




\begin{tabular}{ccccccc} 
& K-S & 0.16 & 0 & 0.03 & 0 & 0 \\
\hline $\begin{array}{c}\text { Out-of- } \\
\text { plane }\end{array}$ & t-test & 0.42 & 0 & 0.66 & 0 & 0 \\
FOD & KWW & 0.27 & 0 & 0.83 & 0 & 0 \\
& 0.1 & 0 & 0 & 0 & 0
\end{tabular}

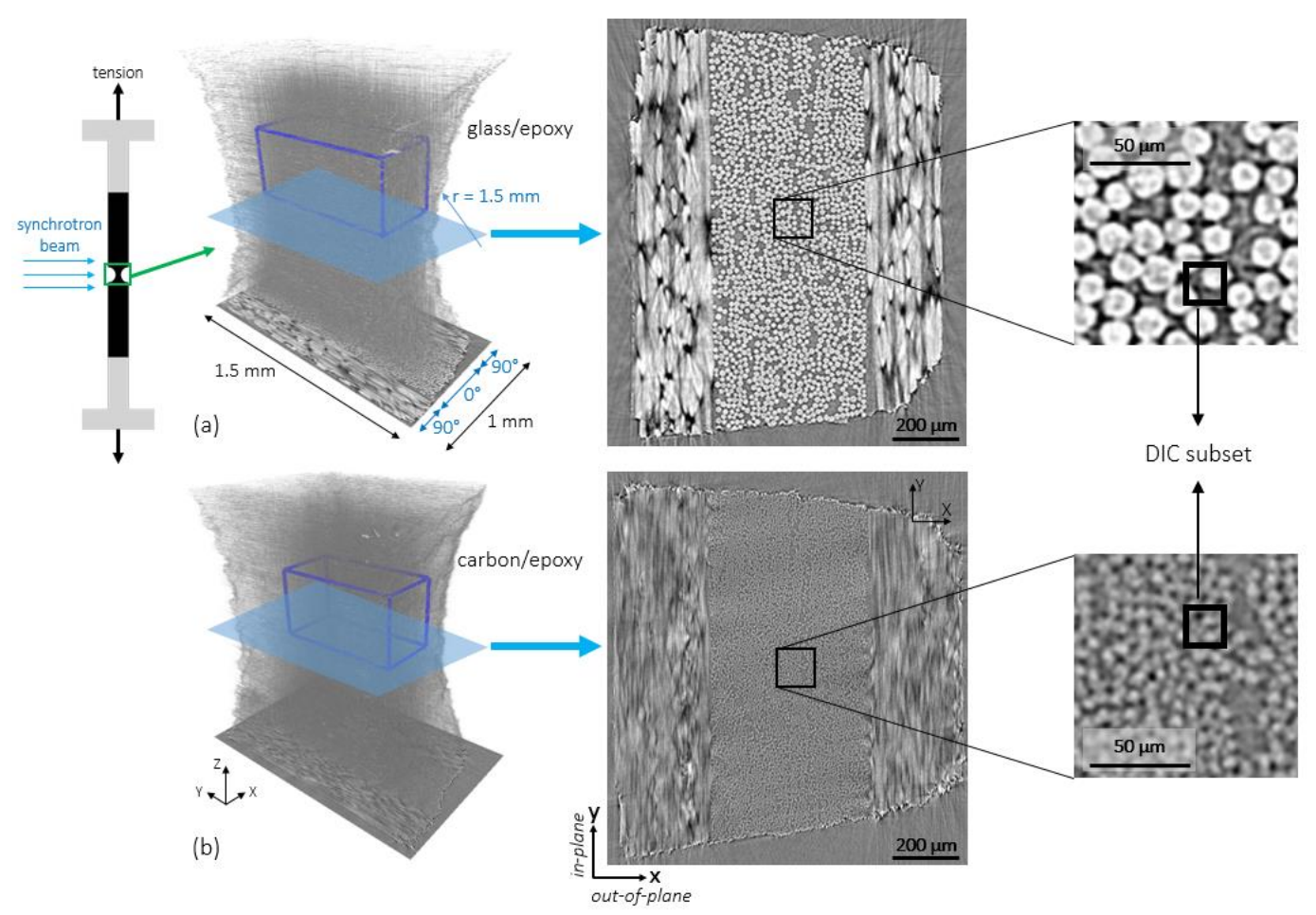

Figure 1. Volume rendering representation and the mid-length $y x$ slice acquired via synchrotron scanning of (a) the glass/epoxy and (b) carbon/epoxy cross-ply laminates - the blue boxes indicate the volume of interests used for fiber orientation analysis. 

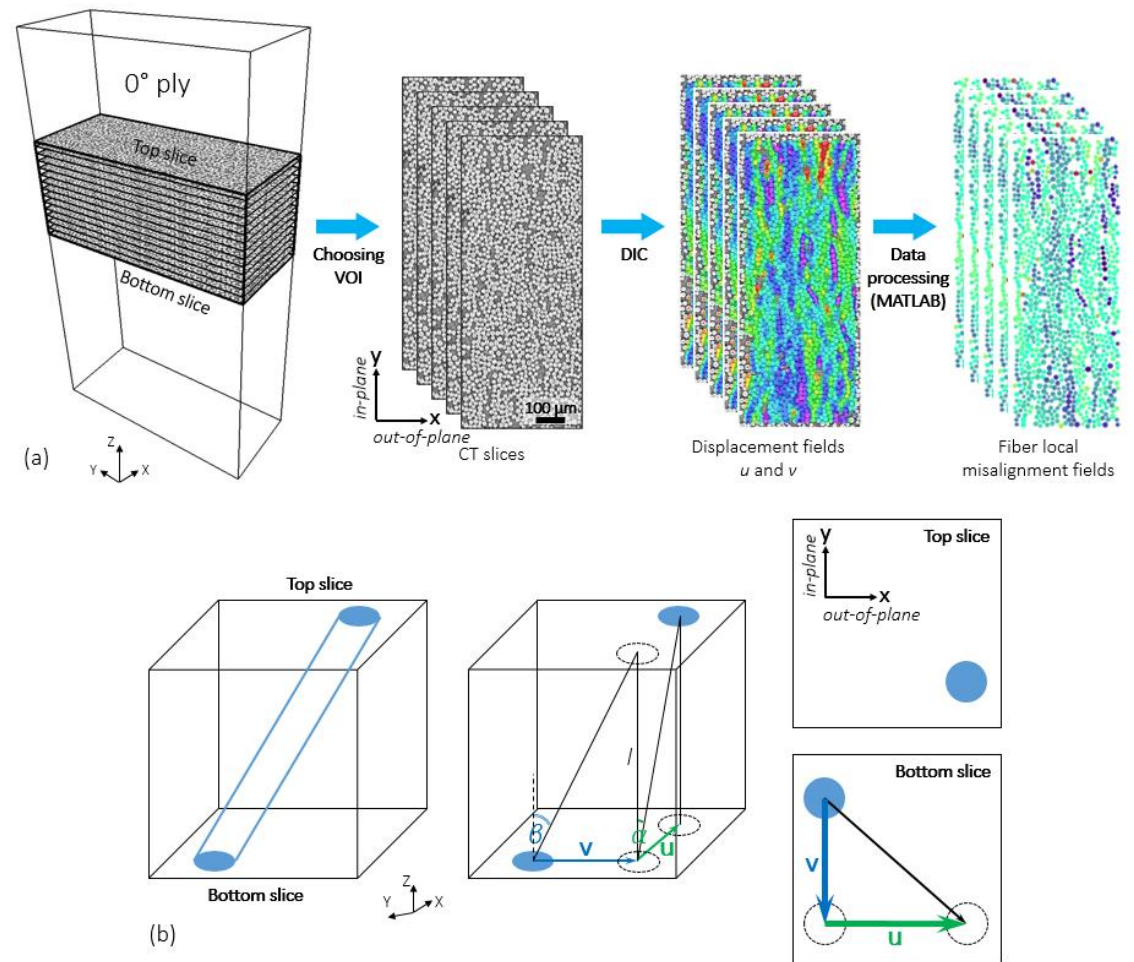

Figure 2. (a) The roadmap of DIC orientation measurement technique, demonstrated on example slices of the glass VOI, and (b) schematics explaining the measurement of inplane and out-of-plane displacements and their translation to corresponding orientations.

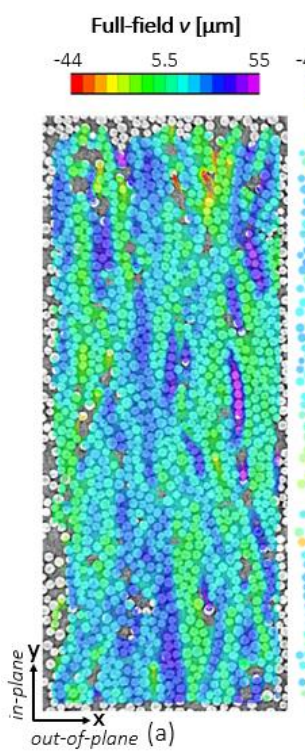

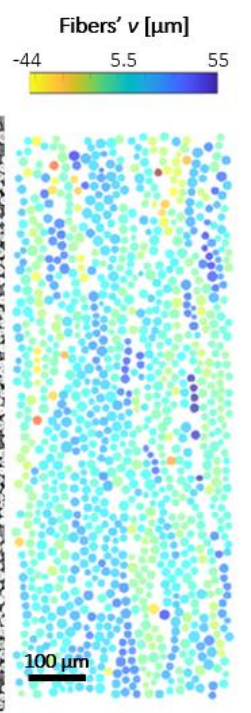

(b)

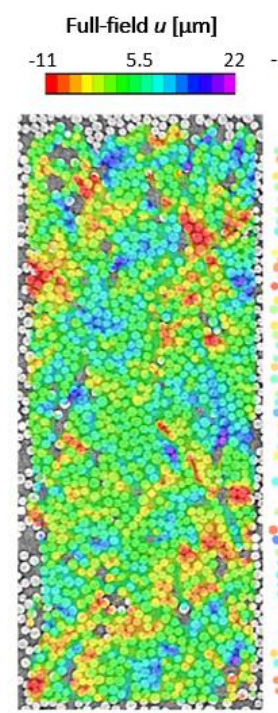

(c)
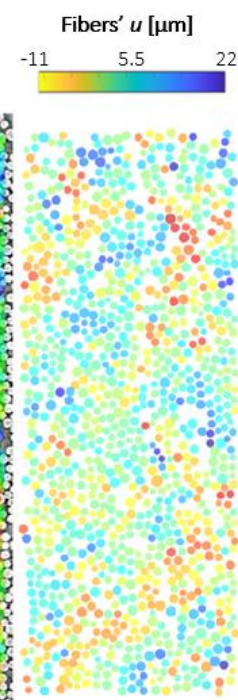

(d) displacement

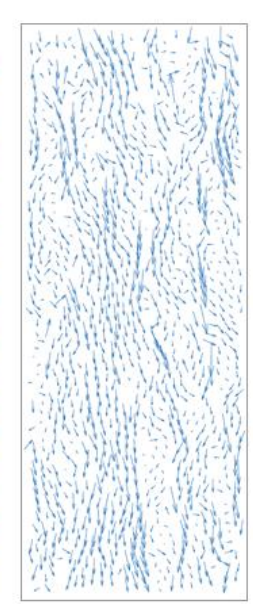

(e)

Figure 3. (a) and (c): DIC displacement fields of the last slice of the undeformed glass VOI (as an example); (b) and (d): DIC-resulting displacement for the detected fibers in the same last slice, extracted from the corresponding displacement field in MATLAB; (e) The displacement vector of the detected fibers displayed on the location of the fibers in the last slice. 

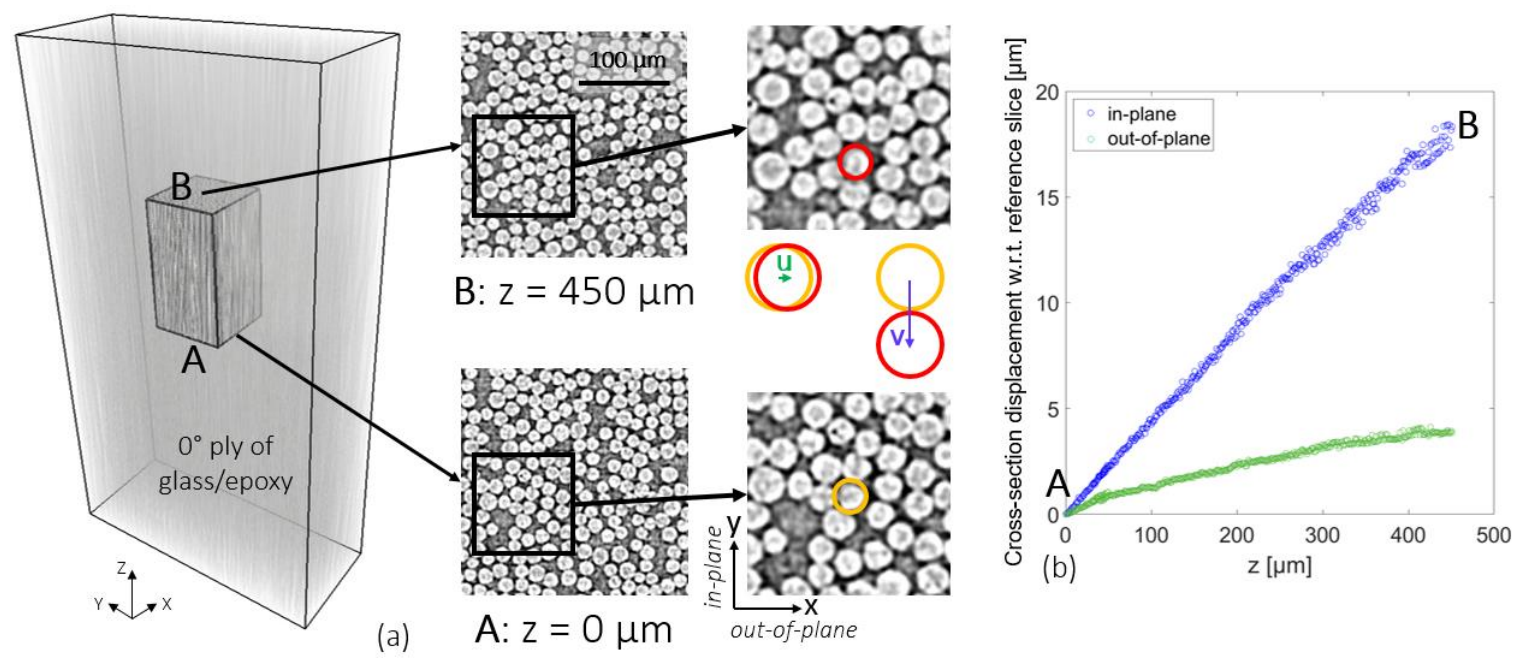

Figure 4. (a) Demonstration and manual verification of the methodology on one fiber in the glass/epoxy material; (b) DIC in-plane and out-of-plane displacements of that fiber in each slice, measured with regard to the first reference slice. The manually measured inplane and out-of-plane displacements in the last slice are 18.7 and $4.4 \mu \mathrm{m}$, while the DIC resulting values are 18.3 and $3.9 \mu \mathrm{m}$, respectively.
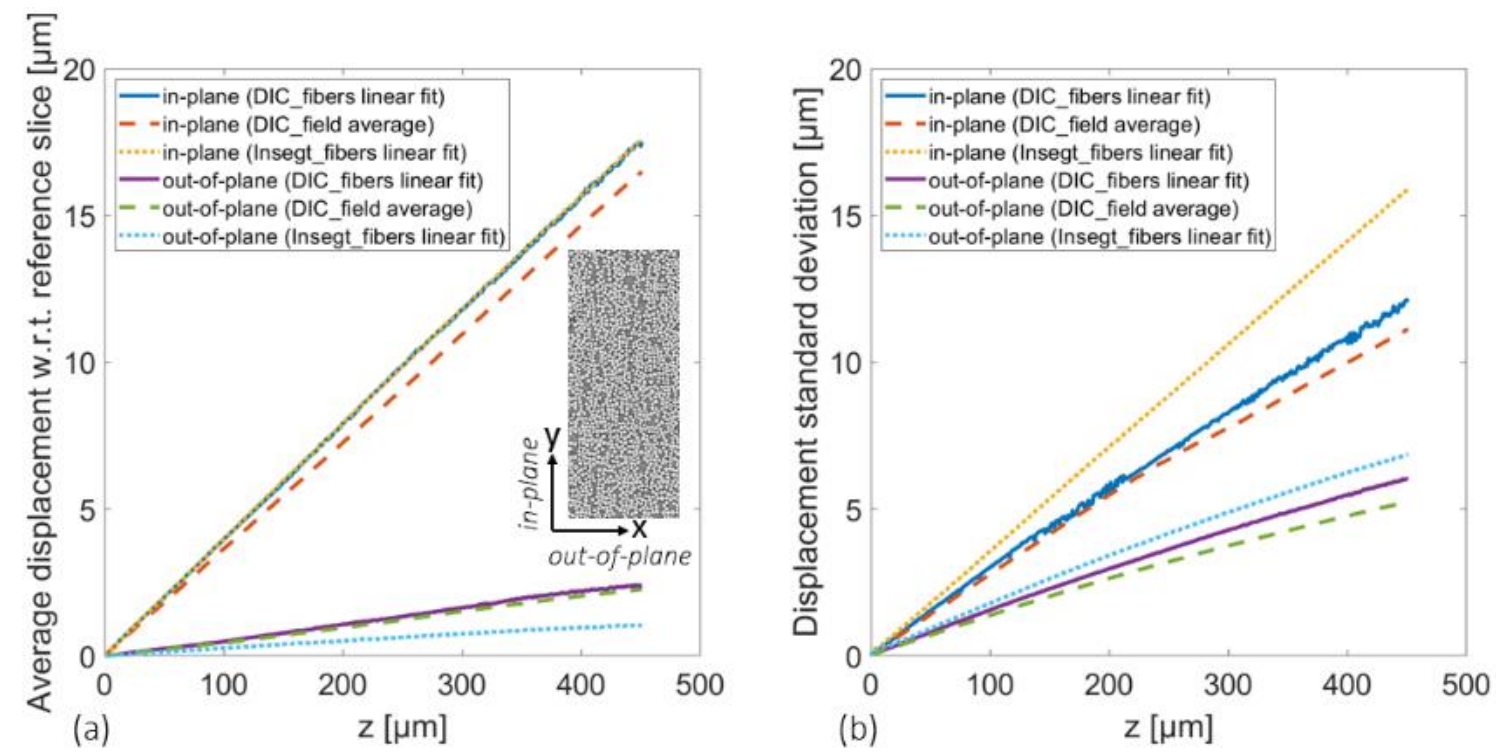

Figure 5. (a) and (b): Average and standard deviation of displacement of all fibers in every slice of the glass VOI plotted versus the slice $z$-coordinate, compared with the average and standard deviation of the entire DIC displacement fields and with the average and standard deviation of displacement of all fibers measured via Insegt Fibre. 

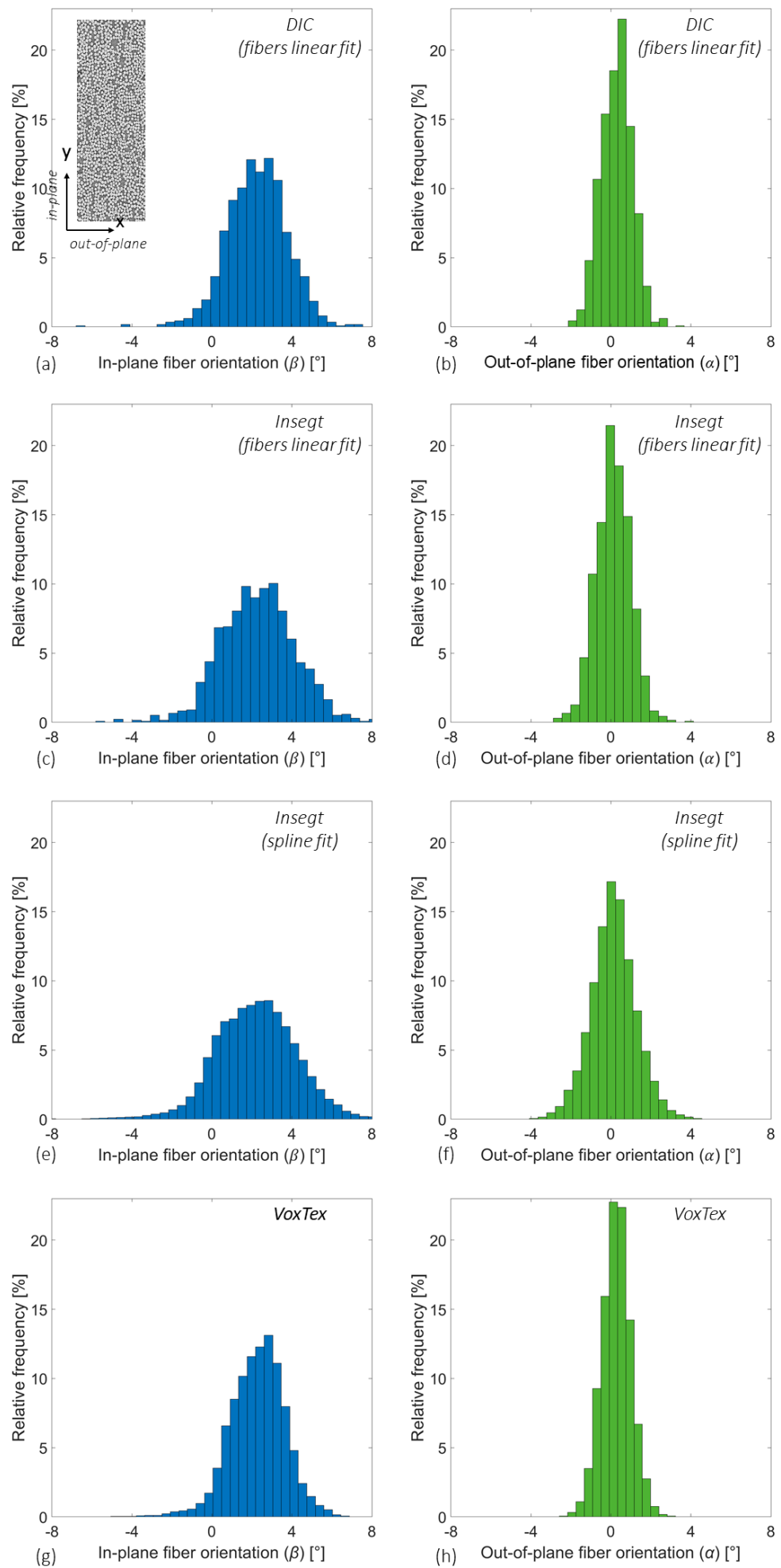

Figure 6. (a) and (b): DIC resulting FODs in the glass VOI, of which the orientations are calculated using the slope of the line that fits the fiber location (or alternatively their displacements) through successive slices; (c) and (d): Insegt Fibre FODs, obtained similarly to the DIC distributions; (e) and (f): Insegt Fibre FODs, obtained via plotting the local orientation of the 11- $\mu \mathrm{m}$ splines used in fitting the fibers; $(\mathrm{g})$ and $(\mathrm{h})$ : VoxTex resulting FODs. 

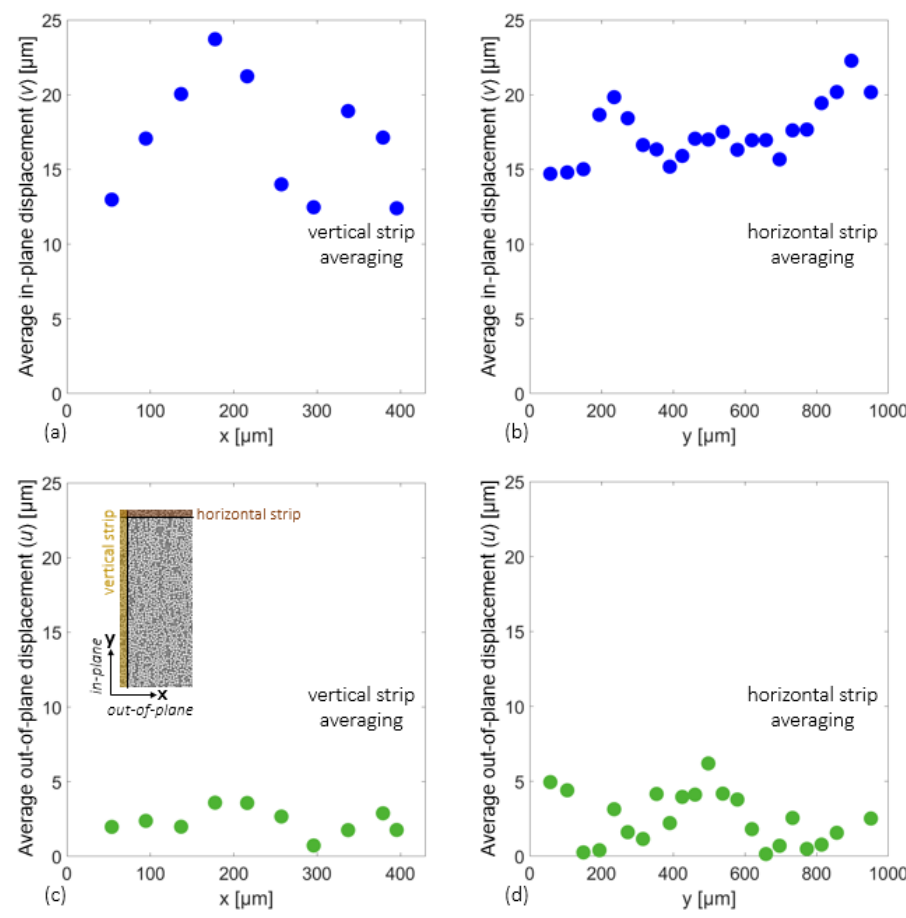

Figure 7. (a) and (b) In-plane and (c) and (d) out-of-plane average displacements, over both vertical and horizontal strips of $40-\mu \mathrm{m}$ width (displayed on (c)) in the last slice of the glass VOI.
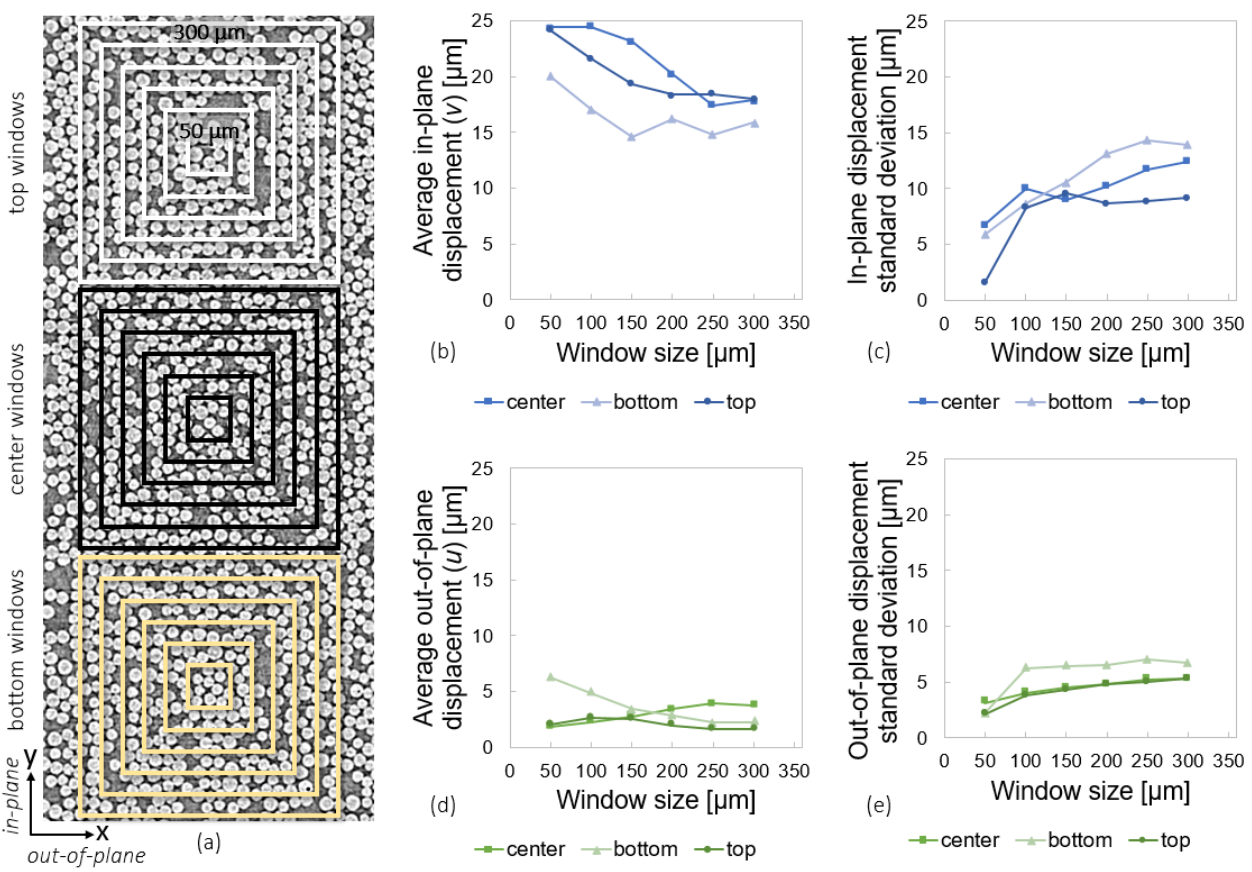

Figure 8. Misalignment RVE size analysis for the glass VOI: (a) windows of different sizes within 50-300 $\mu \mathrm{m}$ at three different locations along the width of the VOI; (b)-(e): the average and standard deviation of the displacements in these windows. 

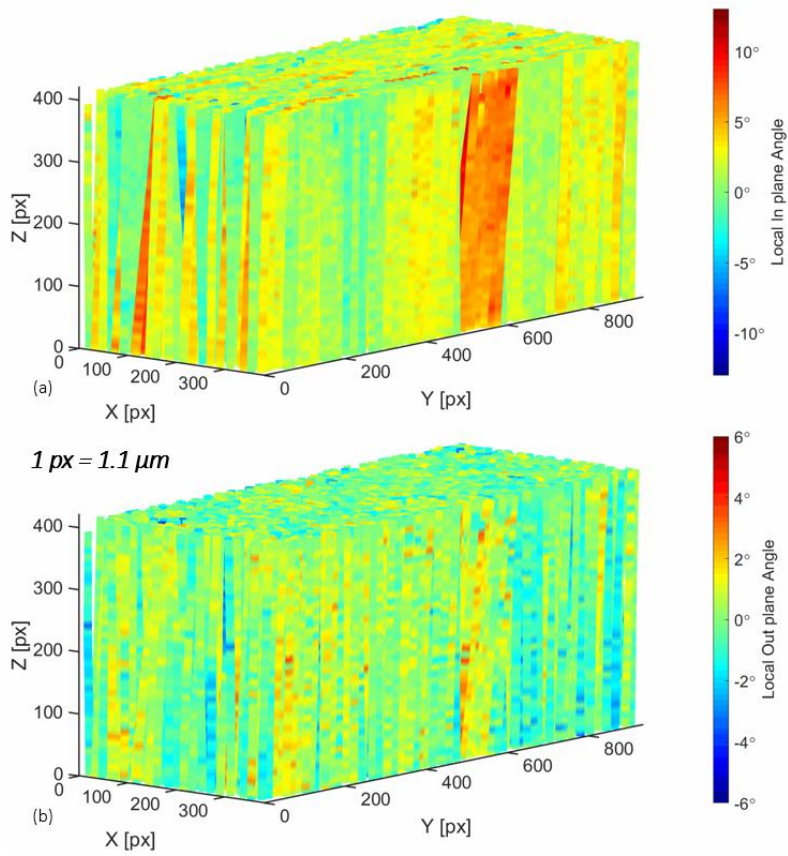

Figure 9. Fiber trajectories in the glass VOI, tracked by Insegt Fibre, smoothed over segments of $11 \mu \mathrm{m}$, and sampled with $11-\mu \mathrm{m}$ splines. The trajectories are visualized with a diameter of $15 \mu \mathrm{m}$ and color coded with (a) in-plane and (b) out-of-plane orientations.

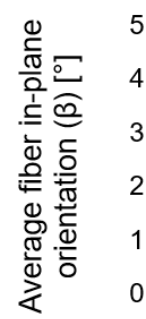

(a)
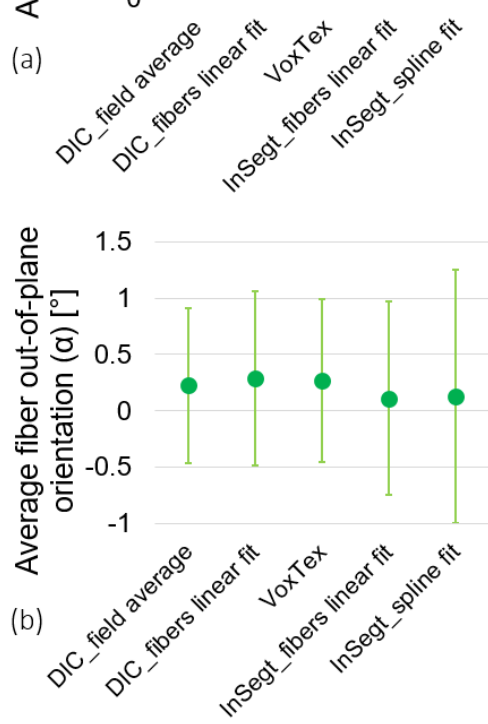

Figure 10. The averages and standard deviations of fiber orientations in the glass VOI, calculated with different methods. The error bars on each data point represent the corresponding standard deviation for that method. 

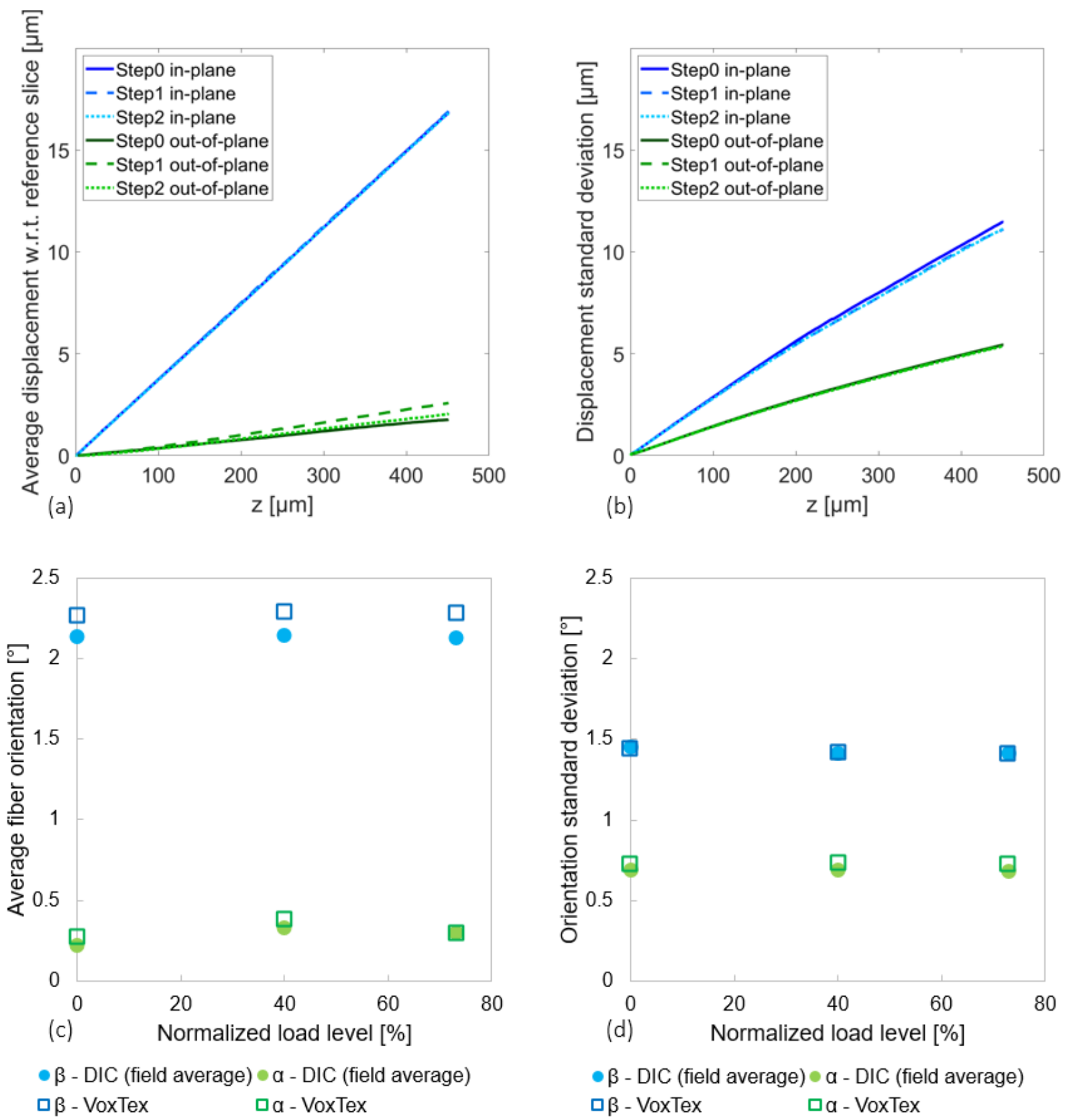

$\bullet \beta$ - DIC (field average) $\propto \alpha$ - DIC (field average)

$\square \beta-$ VoxTex

$\square$ a - VoxTex

$$
\square \beta \text { - VoxTex } \square \text { a-VoxTex }
$$

Figure 11. (a) and (b): The averages and standard deviations of DIC in-plane and out-ofplane displacements in each slice versus the slice $z$-coordinate, for the three consecutive loading steps of the glass VOI; (c) and (d): the average and standard deviation of fiber orientations, calculated using the slope of the line passing through the first and last data points of the curves in (a) and (b) versus the corresponding averages and standard deviations measured via VoxTex $-\alpha$ and $\beta$ are out-of-plane and in-plane misalignment, respectively. 


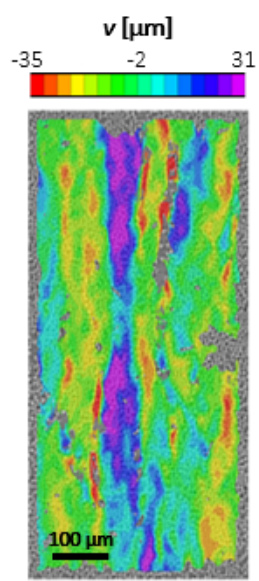

(a)

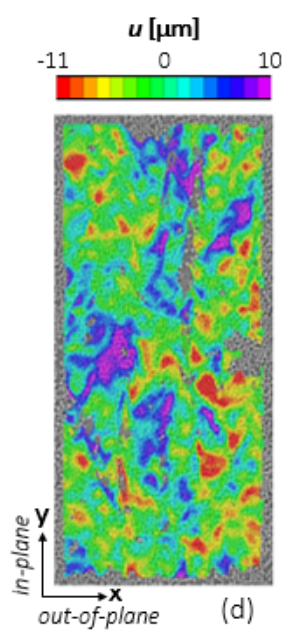

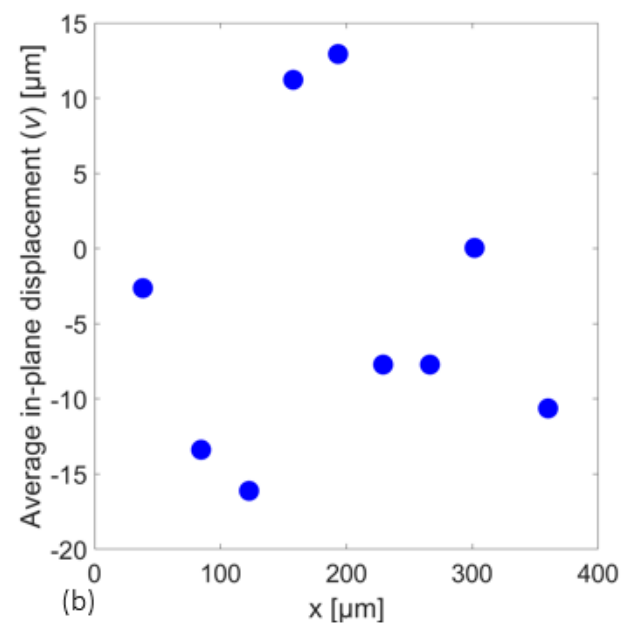

(b)

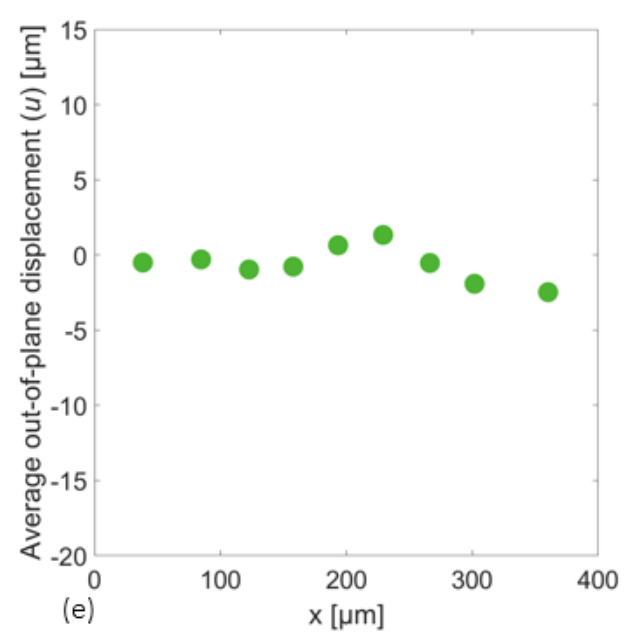

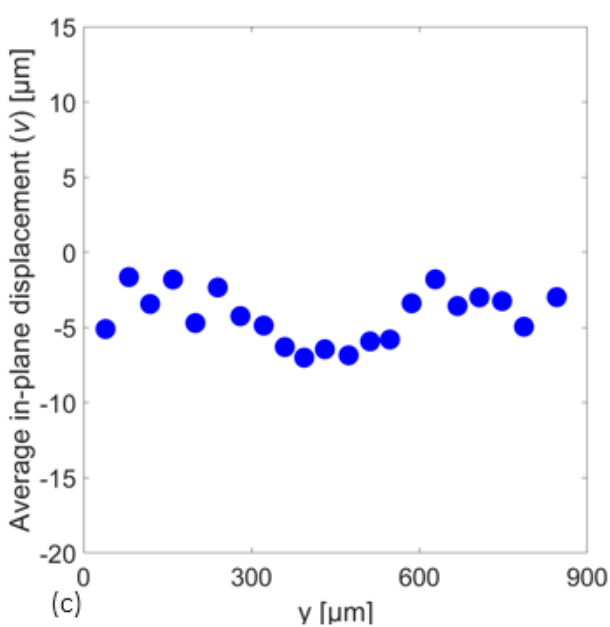

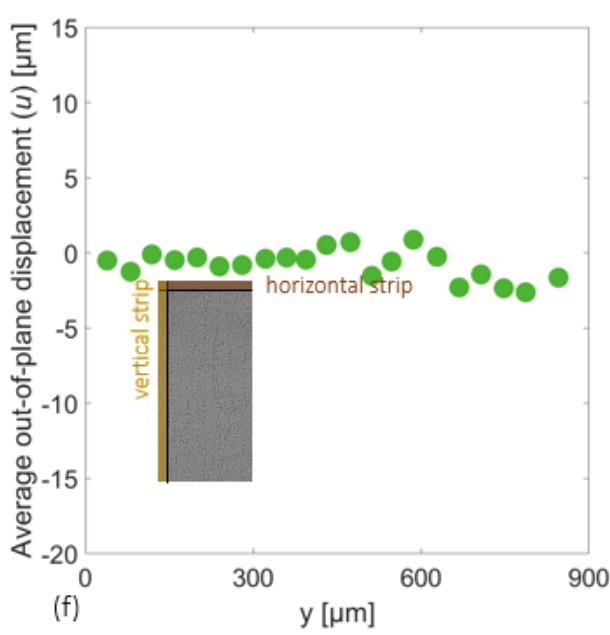

Figure 12. (a) and (d): DIC displacement fields of the last slice of the undeformed carbon VOI; (b), (c), (e), and (f): average displacements over vertical and horizontal strips of 40$\mu \mathrm{m}$ width in the last slice (measured from the DIC field data and not from the individual fibers data). 

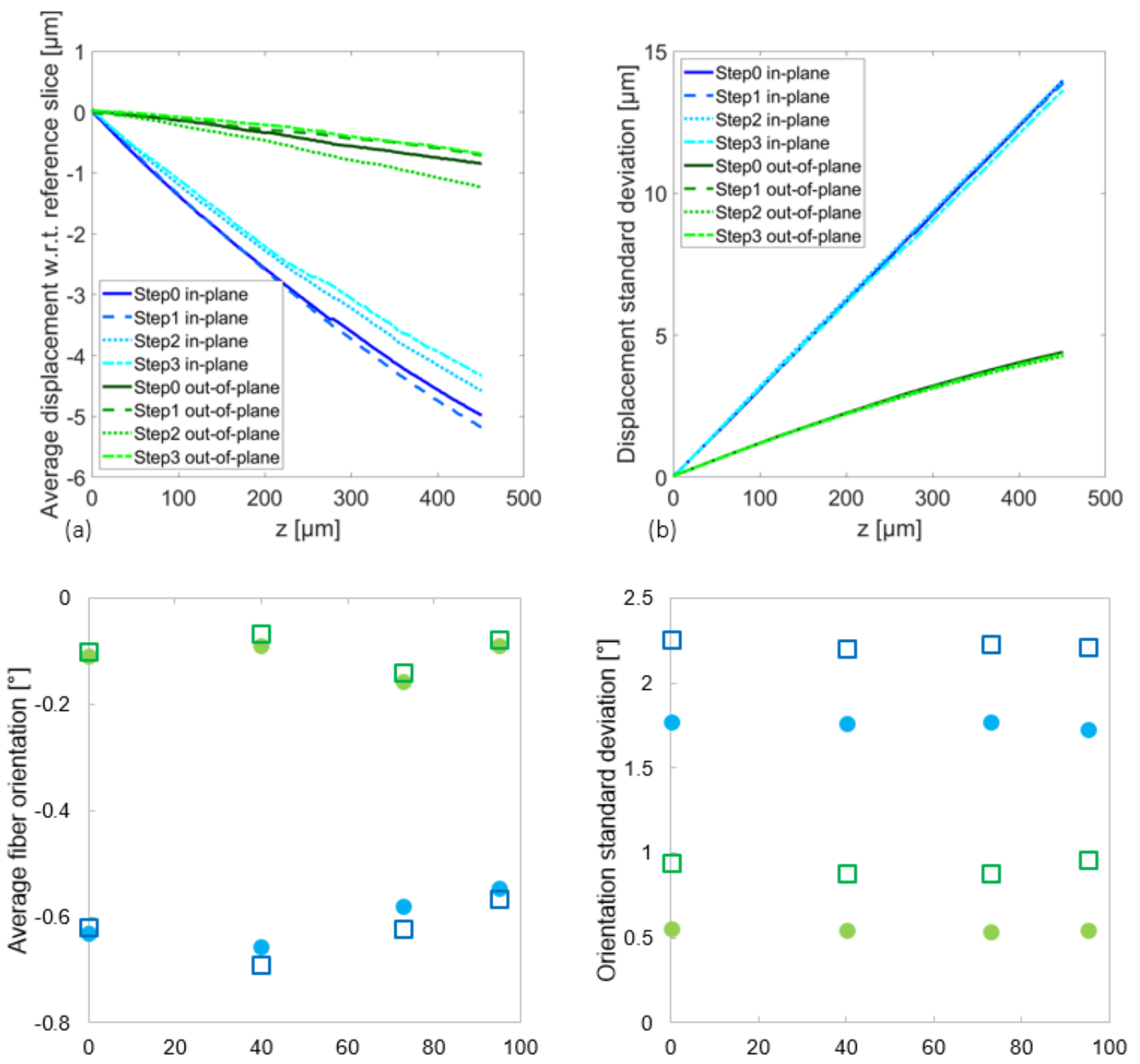

(c) Normalized load level [\%]

(d) Normalized load level [\%]

- $\beta$ - DIC (field average) $\propto \alpha$ - DIC (field average) $\square \beta$-VoxTex $\quad \square \alpha$ - VoxTex

$-\beta$ - DIC (field average) $\propto \alpha$ - DIC (field average) $\square \beta$-VoxTex $\quad \square \alpha-$ VoxTex

Figure 13. (a) and (b): The averages and standard deviations of DIC in-plane and out-ofplane displacements in each slice versus the slice $z$-coordinate, for the four consecutive loading steps of the carbon VOI; (c) and (d): the average and standard deviation of fiber orientations, calculated using the slope of the line passing through the first and last data

points of the curves in (a) and (b) versus the corresponding averages and standard deviations measured via VoxTex $-\alpha$ and $\beta$ are in-plane and out-of-plane misalignment, respectively. 